EPJ Web of Conferences 38, 04003 (2012)

DOI: $10.1051 /$ epjconf/20123804003

(C) Owned by the authors, published by EDP Sciences, 2012

\title{
Self-consistent pairing interaction and collective motion in nuclei: a semiclassical approach
}

\author{
V. I. Abrosimov ${ }^{1}$, D.M. Brink ${ }^{2}$, A. Dellafiore $^{3}$, and F. Matera ${ }^{3}$ \\ ${ }^{1}$ Institute for Nuclear Research, Kiev, Ukraine \\ ${ }^{2}$ Oxford University, Oxford, U.K. \\ ${ }^{3}$ Istituto Nazionale di Fisica Nucleare and Universita di Firenze, Firenze, Italy
}

\begin{abstract}
The solutions of the semiclassical time-dependent Hartree-Fock-Bogoliubov equations of motion are studied in a linear approximation in which the pairing field is allowed to oscillate and to become complex. The pairing field fluctuations are derived from the self-consistent relation (the gap equation of the BCS type), while the static pairing field is approximated with a phenomenological constant $\Delta$. The self-consistent pairingfield fluctuations introduce possibility of new collective modes of the system. We have found out the dispersion relation which determines possible collective modes of the system generated by the pairing interaction. The obvious solution of our dispersion relation at $\omega=0$ corresponds to the Anderson-GoldstoneNambu mode and is related to gauge symmetry. The solutions of dispersion relation have been studied in a simple model, in which nuclei are represented as homogeneous spheres of symmetric nuclear matter characterized by parameters (size, density, pairing gap) typical of heavy nuclei. We have found that the dispersion relation has approximate solution at $\hbar \omega \approx 2 \Delta$ for the monopole channel. Our semiclassical approach is valid for both weak and strong pairing. Here we focus on weak pairing which is suitable for systems with size and pairing parameter appropriated to nuclei.
\end{abstract}

\section{Introduction}

A great amount of work has been done on the effects of pairing in nuclei (see, e. g. [1] and references therein), however there are problems which deserve further attention, especially when finite-size effects become important. One of these topics, which is of current interest both in the physics of nuclei [2] and of other mesoscopic systems [3], is the issue of possible collective phenomena associated with the pairing interaction. Here we address this problem by using a semiclassical approximation for the equations of motion, but taking into account the finite size of the many-body system.

The semiclassical approximation is valid for small or mesoscopic Fermi systems with a characteristic size $R$, provided $p_{F} R / \hbar>>1$, where $p_{F}$ is the Fermi momentum. This is equivalent to the condition that $\hbar \omega_{0}<<\varepsilon_{F}$, where $\omega_{0}$ is a frequency characteristic of the classical orbits and $\varepsilon_{F}$ is the Fermi energy. Since in heavy nuclei the condition $p_{F} R / \hbar>>1$ is well satisfied, in [4,5] we have used an approach similar to that of Ref. [6] to study the linear response of (heavy) nuclei, with the aim of developing a simplified tool for the study of pairing effects in collective nuclear excitations.
Here the pairing interaction is included in semiclassical equations of motion as in Refs. [4-6]. We focus on weak pairing where the pairing gap $\Delta<<\hbar \omega_{0}$. Our approach is concerned with the frequency response of nuclei and in our formalism the pairing gap is a frequency. The pairing gap may be small, while the frequency $\Delta / \hbar$ remains finite in the formal limit $\hbar \rightarrow 0$. Mesoscopic systems with $\Delta>>\hbar \omega_{0}$ can also be studied with the formalism of this paper, but they are not discussed here.

We use the improved linear approximation in which the pairing field $\Delta(\mathbf{r}, \mathbf{p}, t)$ is allowed to oscillate and to become complex [5]. In present approach the static pairing field $\Delta_{0}(\mathbf{r}, \mathbf{p})$ is approximated with a real, constant, phenomenological parameter $\Delta$, hence our approach is not fully self-consistent, however, the (complex) pairingfield fluctuations $\delta \Delta(\mathbf{r}, \mathbf{p}, t)$ are derived from selfconsistent relations. We study the new set of equations of motion that arise in this improved approximation and determine the effects of the self-consistent pairing-field fluctuations on the normal density response function of nuclei. The resulting equations allow also for the study of possible collective effects associated with the pairing interaction. 
In section 2, the dynamical equations with pairing for small amplitudes are formulated in terms of the normal and anomalous phase-space distribution functions. In section 3, the self-consistency condition for the pairingfield fluctuations is derived from the gap equation of the BCS type. We introduce the approximations concerning the self-consistency condition that allow us to reduce the problem of solving of the integral relations to the solution of a system of coupled algebraic equations. In section 4, the solution of the dynamical equations with pairing for finite systems is studied by using the linear response function formalism and the method of action-angle variables. We consider the effects of the self-consistent pairing-field fluctuations on collective motion in heavy nuclei. Finally, in section 5 conclusions are drawn.

\section{Dynamical equations with pairing}

We use the semiclassical equations of motion for the time-dependent Hartree-Fock-Bogoliubov problem in a linear approximation as dynamical equations. We assume that our system is saturated both in spin and isospin space, so we do not need to introduce explicitly these variables. Thus dynamical equations of our approach can be written as [5]

$$
\begin{aligned}
& i \hbar \partial_{t} \delta \rho^{e v}=i \hbar\left\{h_{0}, \delta \rho^{o d}\right\}-i 2\left(\Delta \delta \kappa^{i}-\kappa_{0} \delta \Delta^{i}\right), \\
& i \hbar \partial_{t} \delta \rho^{o d}=i \hbar\left\{h_{0}, \delta \rho^{e v}\right\}+i \hbar\left\{\delta h, \rho_{0}\right\}+i \hbar\left\{\delta \Delta^{r}, \kappa_{0}\right\} \\
& i \hbar \partial_{t} \delta \kappa=2\left(h_{0}-\mu\right) \delta \kappa-\left(2 \rho_{0}-1\right) \delta \Delta+2 \kappa_{0} \delta h-2 \Delta \delta \rho^{e v}
\end{aligned}
$$

with

$$
\begin{aligned}
& \delta \rho^{e v(o d)}(\mathbf{r}, \mathbf{p}, t)=\frac{1}{2}[\delta \rho(\mathbf{r}, \mathbf{p}, t) \pm \delta \rho(\mathbf{r},-\mathbf{p}, t)] \\
& \delta \kappa(\mathbf{r}, \mathbf{p}, t)=\delta \kappa^{r}(\mathbf{r}, \mathbf{p}, t)+i \delta \kappa^{i}(\mathbf{r}, \mathbf{p}, t) .
\end{aligned}
$$

We have the equations of motion for the variations of the normal phase-space distribution function $\delta \rho(\mathbf{r}, \mathbf{p}, t)$ and the anomalous one $\delta \kappa(\mathbf{r}, \mathbf{p}, t)$ from equilibrium distribution $\rho_{0}(\mathbf{r}, \mathbf{p})$ and $\kappa_{0}(\mathbf{r}, \mathbf{p})$. The variation $\delta \rho(\mathbf{r}, \mathbf{p}, t)$ is a real function while $\delta \kappa(\mathbf{r}, \mathbf{p}, t)$ is a complex one. To first order, $\delta \kappa^{\mathrm{r}}(\mathbf{r}, \mathbf{p}, t)$ gives the change in magnitude of $\kappa(\mathbf{r}, \mathbf{p}, t)$ and $\delta \kappa^{\mathrm{i}}(\mathbf{r}, \mathbf{p}, t)$ is proportional to the change in the phase of $\kappa(\mathbf{r}, \mathbf{p}, t)$.

The two equilibrium phase-space distributions $\rho_{0}(\mathbf{r}, \mathbf{p})$ and $\kappa_{0}(\mathbf{r}, \mathbf{p})$ are given by [7] (note the opposite sign of our function $\kappa_{0}$, compared to the function $\kappa$ in Ref. [7])

$$
\begin{aligned}
& \rho_{0}(\mathbf{r}, \mathbf{p})=\frac{1}{2}\left(1-\frac{h_{0}(\mathbf{r}, \mathbf{p})-\mu}{E(\mathbf{r}, \mathbf{p})}\right), \\
& \kappa_{0}(\mathbf{r}, \mathbf{p})=-\frac{\Delta_{0}(\mathbf{r}, \mathbf{p})}{2 E(\mathbf{r}, \mathbf{p})}
\end{aligned}
$$

with the quasiparticle energy

$$
E(\mathbf{r}, \mathbf{p})=\sqrt{\left(h_{0}(\mathbf{r}, \mathbf{p})-\mu\right)^{2}+\Delta_{0}^{2}(\mathbf{r}, \mathbf{p})} .
$$

The chemical potential $\mu$ is determined by the number of nucleons $A$ through the relation

$$
A=\frac{4}{(2 \pi \hbar)^{3}} \int \mathrm{d} \mathbf{r} \mathrm{d} \mathbf{p} \rho_{0}(\mathbf{r}, \mathbf{p}) .
$$

The equilibrium Hamiltonian

$$
h_{0}(\mathbf{r}, \mathbf{p})=\frac{p^{2}}{2 m}+V_{0}(\mathbf{r})
$$

contains the (Hartree) mean field $V_{0}(\mathbf{r})$, which should be evaluated self-consistently, however in the following we use a phenomenological potential well instead. Like in $[4,5]$, we approximate the static nuclear mean field with a spherical square-well potential of radius $R$, this choice allows us to take into account finite-size effects and, at the same time, to recover the simplicity of homogeneous systems. In the following, we also approximate the equilibrium pairing field $\Delta_{0}(\mathbf{r}, \mathbf{p})$ with the phenomenological parameter $\Delta$ which, in heavy nuclei takes values between 1 and $1.5 \mathrm{MeV}$ [8]. As a consequence of this approximation, both the static and dynamic equations are considerably simplified. The static distributions, in particular, become a function of the particle energy $\varepsilon=h_{0}(\mathbf{r}, \mathbf{p})$ alone, like in an infinite homogeneous system.

The coupled dynamical equations (1)-(3) can be interpreted as an extension of the ordinary linearized Vlasov equation of normal systems to systems with pairing. In the limit $\Delta \rightarrow 0$, the first two equations give the usual linearized Vlasov equation, while the third equation can be ignored. When $\Delta \neq 0$ the three equations (1)-(3) are coupled.

We want to consider the effects of the self-consistent pairing-field fluctuations on the normal density response function of nuclei, thus we assume that our system is initially at equilibrium and that at time $t=0$ it is perturbed by a weak external driving field of the kind

$$
V^{e x t}(\mathbf{r}, t)=\eta \delta(t) Q(\mathbf{r})
$$

Here $\delta(t)$ is a Dirac $\delta$-function in time and $\eta$ is a parameter specifying the strength of the external field. The mean-field fluctuations $\delta h(\mathbf{r}, t)$ can be treated with different degrees of approximation:

$\delta h(\mathbf{r}, t) \approx \delta V^{e x t}(\mathbf{r}, t)$

or

$$
\delta h(\mathbf{r}, t) \approx \delta V^{i n t}(\mathbf{r}, t)+\delta V^{e x t}(\mathbf{r}, t)
$$

The zero-order approximation (12), which corresponds to the quantum single-particle approximation, takes into account only the extra force on a particle due to the driving external field, while in the self-consistent approximation (13) one takes into account 
also changes of the mean field due to the change of the density of surrounding particles. We shall consider only the zero-order approximation for the normal mean-field, while the pairing field is treated more carefully.

We want to study the solution of dynamical equations (1)-(3) for finite Fermi systems taking into account the self-consistent pairing-field fluctuations $\delta \Delta^{\mathrm{r}, \mathrm{i}}(\mathbf{r}, \mathbf{p}, t)$. To meet this end we should define the self-consistent residual pairing interaction.

\section{Self-consistency condition}

In a linear approximation the pairing field $\Delta(\mathbf{r}, \mathbf{p}, t)$ is given as the sum of the equilibrium pairing field $\Delta_{0}(\mathbf{r}, \mathbf{p})$ and a small variation $\delta \Delta(\mathbf{r}, \mathbf{p}, t)$ that is complex, see Eqs. (1)-(3),

$$
\Delta(\mathbf{r}, \mathbf{p}, t)=\Delta_{0}(\mathbf{r}, \mathbf{p})+\delta \Delta^{r}(\mathbf{r}, \mathbf{p}, t)+i \delta \Delta^{i}(\mathbf{r}, \mathbf{p}, t) .
$$

We consider the approximation in which the static pairing field $\Delta_{0}(\mathbf{r}, \mathbf{p})$ is approximated with a phenomenological energy gap $\Delta$, while the pairing field fluctuations $\delta \Delta^{\mathrm{r}, \mathrm{i}}(\mathbf{r}, \mathbf{p}, t)$ are derived from self-consistent relations. Thus, in the present approach, the pairing field is approximated as

$$
\Delta(\mathbf{r}, \mathbf{p}, t) \approx \Delta+\delta \Delta^{r}(\mathbf{r}, t)+i \delta \Delta^{i}(\mathbf{r}, t)
$$

The possible momentum dependence of the complex fluctuations is neglected in order to simplify the theory.

The present approach contains the two extra unknown functions $\delta \Delta^{\mathrm{r}}(\mathbf{r}, t)$ and $\delta \Delta^{\mathrm{i}}(\mathbf{r}, t)$, hence we need two additional equations in order to determine these quantities.

In a self-consistent theory, the changes in the pairing field are related to the changes in the anomalous density $\delta \kappa^{\mathrm{r}, \mathrm{i}}$. There are many possible choices for the selfconsistency relation. Two minimum requirements are that the total particle number should be conserved and that the value of the energy weighted sum rule should be correct (the same as in normal systems). A choice that satisfies both requirements is obtained from the self-consistency relation (gap equation) written in the form $[6,9]$

$$
g \int \frac{d \mathbf{p}}{(2 \pi \hbar)^{3}}\left(\frac{\kappa(\mathbf{r}, \mathbf{p}, t)}{\Delta(\mathbf{r}, t)}+\frac{1}{p^{2} / m}\right)=1 .
$$

Here $g$ is a parameter determining the strength of the pairing interaction. We have assumed that the $p$ dependence of the dynamic pairing field can be neglected. By differentiating Eq. (16), we get the firstorder relation

$$
\int \frac{d \mathbf{p}}{(2 \pi \hbar)^{3}}\left(\delta \kappa(\mathbf{r}, \mathbf{p}, t)-\kappa_{0}(\mathbf{r}, \mathbf{p}) \frac{\delta \Delta(\mathbf{r}, t)}{\Delta}\right)=0,
$$

where $\kappa_{0}(\mathbf{r}, \mathbf{p})$ and $\Delta$ are real equilibrium quantities, while $\delta \kappa(\mathbf{r}, \mathbf{p}, t)$ and $\delta \Delta(\mathbf{r}, t)$ are their complex fluctuations.

The real and imaginary parts of Eq. (17) give the two independent relations

$$
\begin{gathered}
\int d \mathbf{r} \int \frac{d \mathbf{p}}{(2 \pi \hbar)^{3}}\left(\delta \kappa^{r}(\mathbf{r}, \mathbf{p}, t)-\kappa_{0}(\mathbf{r}, \mathbf{p}) \frac{\delta \Delta^{r}(\mathbf{r}, t)}{\Delta}\right)=0, \\
\int d \mathbf{r} \int \frac{d \mathbf{p}}{(2 \pi \hbar)^{3}}\left(\delta \kappa^{i}(\mathbf{r}, \mathbf{p}, t)-\kappa_{0}(\mathbf{r}, \mathbf{p}) \frac{\delta \Delta^{i}(\mathbf{r}, t)}{\Delta}\right)=0 .
\end{gathered}
$$

These conditions, based on Eq. (16) take into account the residual pairing interaction in a self-consistent way. Note that, since the conditions (18)-(19) do not depend on $g$, they are valid both for weak and strong pairing. For our purpose, we do not need further information about the pairing interaction.

Using Eq. (7) in the constant- $\Delta$ approximation gives

$$
\begin{aligned}
& \int d \mathbf{r} \int \frac{d \mathbf{p}}{(2 \pi \hbar)^{3}}\left(\delta \kappa^{r}(\mathbf{r}, \mathbf{p}, t)+\frac{\delta \Delta^{r}(\mathbf{r}, t)}{2 E(\varepsilon)}\right)=0 \\
& \int d \mathbf{r} \int \frac{d \mathbf{p}}{(2 \pi \hbar)^{3}}\left(\delta \kappa^{i}(\mathbf{r}, \mathbf{p}, t)+\frac{\delta \Delta^{i}(\mathbf{r}, t)}{2 E(\varepsilon)}\right)=0 .
\end{aligned}
$$

Self-consistency conditions similar to these have been used by the authors of Ref. [3] in their quantum calculations for infinite homogeneous systems.

The two integral relations (18)-(19), together with the four equations of motion (1)-(3), are not sufficient to determine a unique solution of our problem; hence we need to introduce a further assumption, or 'Ansatz': we assume that the pairing field fluctuations are proportional to the fluctuations of the mean field, that is

$\delta \Delta^{r, i}(\mathbf{r}, \omega)=\eta R^{r, i}(\omega) \delta h(\mathbf{r}, \omega)$

The two response functions $R^{\mathrm{r}, \mathrm{i}}(\omega)$ can be determined from the equations of motion and from the selfconsistency integral relations. In the following we do this within the zero-order approximation (12), that is, we take $\delta h(\mathbf{r}, \omega)=\eta Q(\mathbf{r})$.

Instead of the self-consistency relations (17), it is more convenient to use the weaker relations

$\int d \mathbf{r} \int \frac{d \mathbf{p}}{(2 \pi \hbar)^{3}}\left(\delta \kappa(\mathbf{r}, \mathbf{p}, t)+\frac{\delta \Delta(\mathbf{r}, t)}{2 E(\varepsilon)}\right)=0$,

that follows immediately from (17).

The approximations (21) and (22) concerning the selfconsistency relations make it possible to reduce the problem of solving of the integral relations to the solution of a system of coupled algebraic equations.

\section{Solution of dynamical equations}

The equations of motion (1)-(3) together with the selfconsistency relations (18)-(19) can be solved for finite systems with the method of action-angle variables $[4,10]$. By using these solutions we study the effects of a selfconsistent pairing interaction on the normal density response function and pairing collective modes. 


\subsection{Action-angle variables}

The method of action-angle variables has proved to be very useful for solving the Vlasov equation in normal systems [10-11], hence we use the same method also for systems with pairing correlations. The action-angle variables $(\mathbf{I}, \Phi)$ have a very useful property. Any onevalued function $F(\mathbf{r}, \mathbf{p})$ of the state of the system, expressed in terms of the canonical variables $(\mathbf{r}, \mathbf{p})$, is a periodic function of the angle variables $\Phi$, and its period in each variable is $2 \pi$. It can be expanded as a multiple Fourier series:

$F(\mathbf{r}, \mathbf{p})=\sum_{\mathbf{n}} F_{\mathbf{n}}(\mathbf{I}) e^{i \mathbf{n} \Phi}$

in our case the vector $\mathbf{n}$ has three integer components $\mathbf{n}$ $=\left(n_{1}, n_{2}, n_{3}\right)$. In principle the sum over the components $n_{i}$ runs from $-\infty$ to $+\infty$, however, in practice it is sufficient to include only a few terms around $n_{\mathrm{i}}=0$, moreover, for spherical systems, the vector $\mathbf{n}$ is effectively twodimensional.

First of all, we take the Fourier transform in time of the equations of motion (1)-(3). Since dynamical quantities are non-vanishing only for $t>0$, we suppose that $\omega$ has a vanishingly small positive imaginary part $i e$, to insure convergence of the integrals. In the following, the imaginary part of $\omega$ will not be written explicitly.

We introduce the following Fourier expansions based on the method of action-angle variables:

$$
\begin{aligned}
& \delta h(\mathbf{r}, \omega)=\sum_{\mathbf{n}} \delta h_{\mathbf{n}}(\mathbf{I}, \omega) e^{i \mathbf{n} \Phi}, \\
& \delta \rho^{e v(o d)}(\mathbf{r}, \mathbf{p}, \omega)=\sum_{\mathbf{n}} \delta \rho_{\mathbf{n}}^{e v(o d)}(\mathbf{I}, \omega) e^{i \mathbf{n} \Phi}, \\
& \delta \boldsymbol{\kappa}^{r, i}(\mathbf{r}, \mathbf{p}, \omega)=\sum_{\mathbf{n}} \delta \kappa_{\mathbf{n}}^{r, i}(\mathbf{I}, \omega) e^{i \mathbf{n} \Phi}, \\
& \delta \Delta^{r, i}(\mathbf{r}, \omega)=\sum_{\mathbf{n}} \delta \Delta_{\mathbf{n}}^{r, i}(\mathbf{I}, \omega) e^{i \mathbf{n} \Phi} .
\end{aligned}
$$

Moreover we use the relation

$$
\left\{f, h_{0}\right\}=\sum_{\mathbf{n}} i \omega_{\mathbf{n}}(\mathbf{I}) f_{\mathbf{n}}(\mathbf{I}) e^{i \mathbf{n} \Phi}
$$

where $\omega_{\mathbf{n}}(\mathbf{I})=\Sigma_{\alpha} \mathrm{n}_{\alpha} \omega_{\alpha}$ are the eigenfrequencies of the uncorrelated system [10], to obtain from the dynamic equations (1)-(3) the system of algebraic equations

$$
\begin{aligned}
& \hbar \omega \delta \rho_{\mathbf{n}}^{e v}(\mathbf{I}, \omega)=\hbar \omega_{\mathbf{n}} \delta \rho_{\mathbf{n}}^{o d}-2 i \Delta\left[\delta \kappa_{\mathbf{n}}^{i}+\frac{\delta \Delta_{\mathbf{n}}^{i}}{2 E(\varepsilon)}\right] \\
& \hbar \omega \delta \rho_{\mathbf{n}}^{o d}(\mathbf{I}, \omega)=\hbar \omega_{\mathbf{n}} \delta \rho_{\mathbf{n}}^{e v}-\frac{d \rho_{o}}{d \varepsilon} \hbar \omega_{\mathbf{n}} \delta h_{\mathbf{n}}-\frac{d \kappa_{o}}{d \varepsilon} \hbar \omega_{\mathbf{n}} \delta \Delta_{\mathbf{n}}^{r},
\end{aligned}
$$

$$
i \hbar \omega \delta \kappa_{\mathbf{n}}^{i}(\mathbf{I}, \omega)=2(\varepsilon-\mu)\left[\delta \kappa_{\mathbf{n}}^{r}+\frac{\delta \Delta_{\mathbf{n}}^{r}}{2 E(\varepsilon)}\right]+2 \kappa_{0} \delta h_{\mathrm{n}}-2 \Delta \delta \rho_{\mathbf{n}}^{e v},
$$

$$
-i \hbar \omega \delta \kappa_{\mathbf{n}}^{r}(\mathbf{I}, \omega)=2(\varepsilon-\mu)\left[\delta \kappa_{\mathbf{n}}^{i}+\frac{\delta \Delta_{\mathbf{n}}^{i}}{2 E(\varepsilon)}\right]
$$

In order to simplify the formulae, here and in the rest of this section we shall not always write explicitly the $\mathbf{I}, \varepsilon$ or $\omega$ dependence of the various quantities.

By using this set of coupled algebraic equations, we can express the quantities $\delta \kappa^{r, i}$ and $\delta \rho^{e v, o d}$ in terms of the pairing field fluctuations $\delta \Delta^{r, i}$ and $\delta V^{e x t}$.

The expressions for the quantities $\delta \kappa^{r, i}$ in terms of the pairing field fluctuations $\delta \Delta^{r, i}$ allow us to write the weaker self-consistency relations (18)-(19) as

$\left[\int d \mathbf{I} a_{11}(\mathbf{n}, \mathbf{I}, \omega) \delta \Delta_{\mathbf{n}}^{r}(\mathbf{I}, \omega)+\int d \mathbf{I} a_{12}(\mathbf{n}, \mathbf{I}, \omega) \delta \Delta_{\mathbf{n}}^{i}(\mathbf{I}, \omega)\right] \delta_{\mathbf{n}, 0}=$

$\eta \int d \mathbf{I} b_{1}(\mathbf{n}, \mathbf{I}, \omega) Q_{\mathbf{n}}(\mathbf{I}) \delta_{\mathbf{n}, 0}$,

$\left[\int d \mathbf{I} a_{21}(\mathbf{n}, \mathbf{I}, \omega) \delta \Delta_{\mathbf{n}}^{r}(\mathbf{I}, \omega)+\int d \mathbf{I} a_{22}(\mathbf{n}, \mathbf{I}, \omega) \delta \Delta_{\mathbf{n}}^{i}(\mathbf{I}, \omega)\right] \delta_{\mathbf{n}, 0}=$

$\eta \int d \mathbf{I} b_{2}(\mathbf{n}, \mathbf{I}, \omega) Q_{\mathbf{n}}(\mathbf{I}) \delta_{\mathbf{n}, 0}$,

The expressions for the functions $a_{\mathrm{ik}}(\mathbf{n}, \mathbf{I}, \omega)$ and $b_{\mathrm{i}}(\mathbf{n}, \mathbf{I}, \omega)$ are given by Eqs. (79)-(83) of [5].

Using the 'Ansatz' (21), in self-consistency relations (33-34) gives a system of coupled algebraic equations for the response functions $R^{\mathrm{r}, \mathrm{i}}(\omega)$. This system has two kind solutions. If the determinant is non- vanishing, the system of coupled algebraic equations (33)-(34) can be solved, giving

$$
\begin{aligned}
& R^{r}(\omega)=0, \\
& R^{i}(\omega)=\frac{2 \Delta}{i \hbar \omega} .
\end{aligned}
$$

However there are also other solutions corresponding to the roots of vanishing determinant. These solutions give frequencies of collective modes related to pairing (pairing vibrations).

\subsection{Normal density response function}

The solutions (35)-(36) allow us to get an explicit expression for the normal density response function, defined as

$$
R(\omega)=\frac{1}{\eta} \int d \mathbf{r} Q(\mathbf{r}) \delta \rho(\mathbf{r}, \omega),
$$

where $\delta \rho(\mathbf{r}, t)$ is the time Fourier transform of the normal particle density fluctuation.

Using the Fourier expansions (24)-(25) we write the response function (37) in terms of action-angle variables as

$$
R(\omega)=\frac{1}{\eta} \frac{4}{(2 \pi \hbar)^{3}} \sum_{\mathbf{n}} \int d \mathbf{I} Q_{\mathbf{n}}^{*}(\mathbf{I}) \delta \rho_{\mathbf{n}}^{e v}(\mathbf{I}, \omega) .
$$

The expression for the normal distribution function $\delta \rho^{e v}{ }_{n}(\mathbf{I}, \boldsymbol{\omega})$ can be obtained from the set of algebraic 
equations (29)-(32) taking into account the solutions (35)-(36). Thus we get

$\delta \rho_{\mathbf{n}}^{e v}(\mathbf{I}, \omega) \approx-\eta \frac{\left[\omega_{\mathbf{n}}(\mathbf{I})\right]^{2}}{\omega^{2}-\left[\bar{\omega}_{\mathbf{n}}(\mathbf{I})\right]^{2}} \frac{d \rho_{0}}{d \varepsilon} Q_{\mathbf{n}}(\mathbf{I})$

with the eigenfrequencies of the correlated system

$\left[\bar{\omega}_{\mathbf{n}}(\mathbf{I})\right]^{2}=\left[\omega_{\mathbf{n}}(\mathbf{I})\right]^{2}+\Omega^{2}(\mathbf{I})$

where $\omega_{\mathbf{n}}(\mathbf{I})$ are the eigenfrequencies of the uncorrelated system (without pairing) and $\Omega(\mathbf{I})=2 E(\varepsilon) / \hbar$. The eigenfrequencies (40) correspond to quantum twoquasiparticle excitations [5].

By using the semiclassical response function (38)(39) we can check that the variation of the particle number $\delta A$ is vanishing. Moreover the value of the energy weighted sum rule (EWSR) is correct. This is easily checked thanks to a well-known theorem [12], according to which, at large $\omega$,

$\left.R(\omega)\right|_{\omega \rightarrow \infty}=\frac{2 M_{1}}{(\hbar \omega)^{2}}+O\left(\frac{1}{(\hbar \omega)^{4}}\right)$,

where $M_{1}$ is the first moment of the strength function, or EWSR. However, the semiclassical response functions (38)-(39) have no additional poles related to the pairing collective modes.

\subsection{Pairing collective modes}

The self-consistent fluctuations of the pairing-field introduce the possibility of extra collective modes of the system induced by the pairing interaction. The possible frequencies of these collective modes are given by the roots of vanishing determinant of coupled algebraic equations (33)-(34) that can be written as [5]

$$
\omega^{2} K(L, \omega)=0 \text {. }
$$

The dispersion relation (42) has the obvious solution at $\omega=0$ that is related to gauge symmetry [1]. We study the possible solutions of the dispersion relation (42) to establish if, for a system with size, density and pairing parameters typical of heavy nuclei, pairing collective modes are to be expected.

We get an explicit expression of the dispersion relation (42) for the monopole channel by using a simple model of nucleus, in which nucleons are confined to a spherical cavity characterized by parameters typical of heavy nuclei [5]. It reads

$$
K(L=0, \omega)=\left[I_{1}(\omega)\right]^{2}-\left[(\hbar \omega)^{2}-4 \Delta^{2}\right]\left[I_{2}(\omega)\right]^{2}=0
$$

with

$$
\begin{aligned}
& I_{2}(\omega)=\int_{0}^{\infty} d \varepsilon g(\varepsilon) \frac{1}{2 E(\varepsilon)} \frac{1}{(\hbar \omega+i e)^{2}-4 E^{2}(\varepsilon)}, \\
& I_{1}(\omega)=\int_{0}^{\infty} d \varepsilon g(\varepsilon) \frac{\varepsilon-\mu}{E(\varepsilon)} \frac{1}{(\hbar \omega+i e)^{2}-4 E^{2}(\varepsilon)},
\end{aligned}
$$

where $g(\varepsilon)$ is the single-particle level density for the equilibrium mean field. In our model the equilibrium mean field is approximated by a spherical square-well potential of radius $R$ (and infinite depth) so that $g(\varepsilon) \propto \varepsilon^{1 / 2}$. For the monopole channel we put in the external field (11) $Q(\mathbf{r})=\mathrm{r}^{2} Y_{00}(\theta, \varphi)$. The integrals (44)-(45) are the semiclassical versions of similar quantities which have been studied in analogous quantum approaches to the problem of pairing vibrations [1]. In the quantum theory, the integrals (44)-(45) are replaced by sum over discrete nuclear levels.

The possible solutions of Eq. (43) are complex. The real part of the solution is defined by the minimum of the absolute value of the function $K$. In figure 1 the absolute value of the function $K(L=0, \omega)$ is shown for the monopole channel. The deep dent of the quantity $K(L=0, \omega)$ at $\omega \approx 2 \mathrm{MeV}$ means that the dispersion relation (43) has an approximate solution at $\omega \approx 2 \Delta$ (the value $\Delta$ $=1 \mathrm{MeV}$ was used in our calculations).

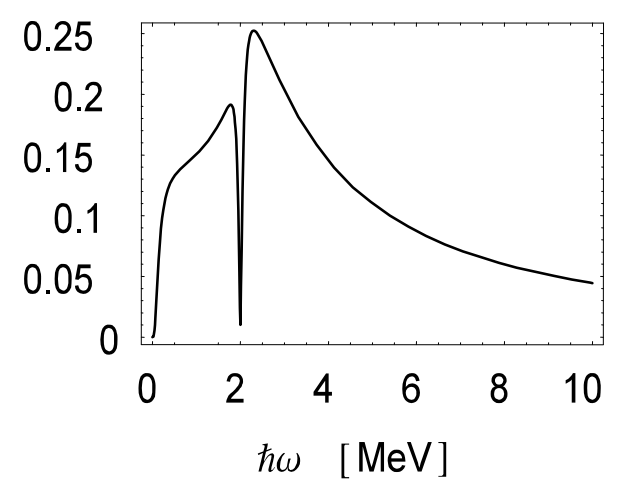

Fig. 1. Absolute value of $K(L=0, \omega)$ for the monopole channel.

It is known that collective pairing modes in nuclei can be strongly excited by two-neutron transfer reactions. To study these processes within the quasiparticle random phase approximation, the response function for the twoparticle transfer is used that is related to the external field which changes the number of particles in the system. It would be of interest to introduce a corresponding external field in our semiclassical approach in order to get the response function that would be more suitable for studying of collective pairing effects.

\section{Conclusions}

We have studied the solutions of the semiclassical timedependent Hartree-Fock-Bogoliubov equations of motion for finite Fermi systems in a linear approximation in which the pairing field is allowed to oscillate and to become complex. The pairing field fluctuations are derived from the self-consistent relations, while the static pairing field is treated in the constant- $\Delta$ approximation. We use our semiclassical approach to study the effects of a self-consistent residual pairing interaction on the isoscalar nuclear density response. 
Within the approximations adopted here, the particlenumber symmetry, which is broken in the constant- $\Delta$ approximation, is restored by the fluctuations of the imaginary pairing field. Moreover all spurious contributions into the density response function are cancelled leading to a correct value of the energyweighted sum rule (the same as for normal systems).

The self-consistent pairing-field fluctuations introduce possibility of new collective modes of the system, generated by the pairing interaction. We have found out the dispersion relation, Eq. (31), which determines possible collective modes of the system induced by the pairing interaction. We found that our semiclassical dispersion relation for pairing vibration in heavy nuclei has approximate solution at $\hbar \omega \approx 2 \Delta$ for the monopole channel, see figure 1.

We have shown that the isoscalar density response of small correlated systems to the external field, which induces small changes of the normal density, see Eq. (13), is suitable to study the effect of pairing correlations on the collective (normal) density excitations, but it is not adequate to investigate collective pairing modes.

Our semiclassical approach is valid for both weak and strong pairing. We focus on weak pairing $\left(\Delta<<\hbar \omega_{\text {coll }}\right)$ which is suitable for systems with size and pairing parameter appropriated to nuclei. It would be of interest to investigate collective excitations of correlated systems with strong pairing where $\varepsilon_{F}>\Delta>>\hbar \omega_{\text {coll }}$ within our semiclassical model. The limit $\Delta>>\hbar \omega_{\text {coll }}$ implies superfluid hydrodynamics so that essential pairing effects on collective motion in finite Fermi systems are expected.

\section{References}

1. D.M. Brink and R.A. Broglia, Nuclear Superfluidity (Cambridge University Press, Cambridge, UK, 2005)

2. W. von Oertzen and A. Vitturi, Rep. Prog. Phys. 64, $1247(2001)$

3. R. Combescot, M. Yu. Kagan, and S. Stringari, Phys. Rev. A 74, 042717 (2006)

4. V.I. Abrosimov, D.M. Brink, A. Dellafiore, and F. Matera, Nucl. Phys. A 800, 1 (2008)

5. V.I. Abrosimov, D.M. Brink, A. Dellafiore, and F. Matera, Nucl. Phys. A 864, 38 (2011)

6. M. Urban and P. Schuck, Phys. Rev. A 73, 013621 (2006)

7. P. Ring and P. Schuck, The Nuclear Many-Body Problem (Springer, N.Y., 1980)

8. A. Bohr and B.R. Mottelson, Nuclear Structure, vol. 1 (Benjamin, N.Y., 1969)

9. T. Papenbrock and G.F. Bertsch, Phys. Rev. C 59, 2052 (1999)

10. D.M. Brink, A. Dellafiore, and M. Di Toro, Nucl. Phys. A 456, 205 (1986)

11. V.L. Polyachenko and I.G. Shukhman, Sov. Astron. 25, 533 (1981)

12. E. Lipparini and S. Stringari, Phys. Rep. 175, 103 (1989) 